\title{
Soft Computing for the Analysis of People Movement Classification
}

\author{
Javier Sedano $^{1}$, Silvia González ${ }^{1}$, Bruno Baruque ${ }^{2}$, Álvaro Herrero ${ }^{2}$, and \\ Emilio Corchado ${ }^{3}$. \\ ${ }^{1}$ Instituto Tecnológico de Castilla y León. C/ López Bravo 70, Pol. Ind. Villalonquejar, \\ 09001 Burgos, Spain. javier.sedano@itcl.es \\ ${ }^{2}$ Civil Engineering Department, University of Burgos. C/ Francisco de Vitoria s/n, 09006 \\ Burgos, Spain. \{bbaruque@ubu.es, ahcosio@ubu.es\} \\ ${ }^{3}$ Departamento de Informática y Automática, University of Salamanca, Plaza de la Merced \\ s/n, 37008 Salamanca, Spain. escorchado@usal.es
}

\begin{abstract}
This article presents a study of the best data acquisition conditions regarding movements of extremities in people. By using an accelerometer, there exist different ways of collecting and storing the data captured while people moving. To know which one of these options is the best one, in terms of classification, an empirical study is presented in this paper. As a soft computing technique for validation, Self-Organizing maps have been chosen due to their visualization capability. Empirical verification and comparison of the proposed classification methods are performed in a real domain, where three similar movements in the real-life are analyzed.
\end{abstract}

\section{Introduction}

Over recent years, the use of Artificial Intelligence (AI) and miniaturized sensors for real world problem solutions has undergone a significant growth.

Some devices integrate sensors to measure movement in the extremities. These are able to record acceleration patterns by means of wearable accelerometer devices, and enable daily physical activity measurement and analysis by applying AI techniques.

Up to now, some solutions have been developed using sensors to measure variables and movement behaviour of the human being, such as the analysis of a cow daily activity data [10], the delivery of patients' information to health care personnel using mobile phones [7], stress detection system [13], etc... 
The use of accelerometers in order to objectively measure body movement is displayed in several studies together with some AI techniques, such as Support Vector Machine (SVM) [3]. Other AI techniques applied for human activity recognition are Hierarchical Hidden Markov Models (HHMM) [16], Fuzzy Basic Functions (FBF) [5], Decision Tree (DT) [8, 3], Neural Network Classifiers such as Resilient Backpropagation (RPROP) [12, 18], K-Nearest Neighbour (K-NN) [4], Dynamic Time Warping (DTW) [11], Kohonen self-organizing maps (SOM) [14], or Naive Bayes (NB) [3]. Most of these techniques use statistical measures like mean, standard deviation, variance, interquartile range, energy, correlation between axes and entropy, for the different features of the data.

This study focuses on determining the way of collecting accelerometer data to improve movement analysis performance. The main point in present study is to find the way of collecting accelerometer data to know the best sampled interval in order to obtain the best divided classes of movements. Thereby, a system could register the data set with the best possible period, optimizing its consumption while improving the performance. On the other hand, this on-going research aims at knowing the number of variables that the model should incorporate to get as low as possible classification error. The final objective is to obtain the furthest classes from the movement data. In order to do so, data are gathered with different time periods and number of variables. Then, in order to obtain some guidelines for the data gathering design, the most relevant group in the data are identified with a SOM.

The remaining sections of this paper are structured as follows: section 2 presents the proposed soft computing approach and the neural projection techniques applied in this work. Some experimental results are presented and described in section 3; the conclusions of this study are discussed in section 4, as well as future work.

\section{Soft-Computing Techniques}

This study proposes to apply soft computing models to people's movement detection. As a first stage, the study aims to investigate which measurement conditions are the best to distinguish correctly the activity in which the user is engaged. To perform this identification automatically, the Self-Organizing Map, has been used. This model was selected because, although: it is originally designed to cluster data samples; but it can also serve to obtain a proper data classification as well. Differentiating from some other models, it can handle unknown or not clearly classifiable data samples and it can also give a visual (and quantifiable) hint of where the new data can be represented with respect of previously presented samples. 


\subsection{Self-Organizing Maps}

Topology preserving mapping comprises a family of techniques with a common target: to produce a low-dimensional representation of the training samples that preserves the topological properties of the input space. From among the various techniques, the best known is the Self-Organizing Map (SOM) algorithm [9].

SOM aims to provide a low-dimensional representation of multi-dimensional data sets while preserving the topological properties of the input space. The SOM algorithm is based on competitive unsupervised learning; an adaptive process in which the neurons in a neural network gradually become sensitive to different input categories, which are sets of samples in a specific domain of the input space. The update of neighbourhood neurons in SOM is expressed as:

$$
\mathrm{w}_{\mathrm{k}}(\mathrm{t}+1)=\mathrm{w}_{\mathrm{k}}(\mathrm{t})+\alpha(\mathrm{t}) \cdot \eta(\mathrm{v}, \mathrm{k}, \mathrm{t}) \cdot\left(\mathrm{x}(\mathrm{t})-\mathrm{w}_{\mathrm{k}}(\mathrm{t})\right)
$$

Where, $x$ denotes the network input, $w_{k}$ the characteristics vector of each neuron; $\alpha$, is the learning rate of the algorithm; and $\eta(v, k, t)$ is the neighbourhood function, in which $v$ represents the position of the winning neuron (Best Matching Unit or BMU) in the lattice, and $k$ the positions of the neurons in its neighbourhood.

\subsection{Quality Measures}

In order to compare the results obtained by the different maps, three of the most widespread measures are used in this study:

- Classification Error [15]. Topology preserving models can be easily adapted for classification of new samples using a semi-supervised procedure. A high value in the classification accuracy rate implies that the units of the map are reacting in a more consistent way to the classes of the samples that are presented. As a consequence, the map should represent the data distribution more precisely.

- Topographic Error [2]. It consists on finding the first two best matching units (BMU) for each entry of the dataset and testing whether the second is in the direct neighbourhood of the first or not.

- Goodness of Map [1]. This measure combines two different error measures: the square quantization error and the grid distortion. It takes account of both the distance between the input and the BMU and the distance between the first BMU and the second BMU in the shortest path between both along the grid map units, calculated solely with units that are direct neighbours in the map. 


\section{Data Gathering and Analysis}

The data collection device consists of a Microchip PIC18F2580 microcontroller and an Analog Device ADXL335 [6] 3D accelerometer [17] that integrates MEMS and SMT technologies. The physical magnitude sampling is performed by the accelerometer and the movement module $(\mathrm{M})$ is calculated on the three axes: $\mathrm{X}, \mathrm{Y}, \mathrm{Z}$.

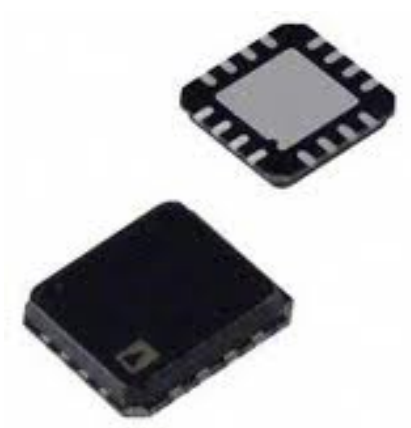

Figure 1. DXL335 Accelerometer.

One female volunteer, aged 25, was enrolled in the study. The accelerometer is placed on the volunteer's right wrists. In this study, a real life dataset was created by performing several activities with similar behaviour in order to provide classes of movements that are very similar. The performed activities have been: walking, walking while holding a bag in the right hand and walking while keeping the right hand into the trousers pocket.

Acceleration vector $-\mathrm{R}(\mathrm{t})$ - was obtained at the beginning of the cycle and subsequently each vector value was collected every $62.5 \mathrm{~ms}$. The values to create the test dataset $(\mathrm{M})$ were calculated in each period of time, with the Euclidean distance [9] between the actual position $-\mathrm{R}(\mathrm{t})$ - and the previous position $-\mathrm{R}(\mathrm{t}-1)-$. Many samples of data from each activity have been created according to different period of time. 2, 4, 8 and 16 data samples from the same activity are grouped by means of simple addition in the data set. For further details check Table 1.

Table 1. Values of the groups.

\begin{tabular}{ll}
\hline $\mathrm{N}^{\mathrm{o}}$ of samples (for each of the 3 classes) & $\mathrm{N}^{\mathrm{o}}$ of measurements grouped per data sample \\
\hline 1000 & $2 / 16$ \\
1000 & $4 / 16$ \\
1000 & $8 / 16$ \\
605 & 1 \\
\hline
\end{tabular}


Finally, three different options for building each of the datasets from these data are considered:

- Option 1 is formed from 4 data sets, each one with two instants of time: previous and current.

- Option 2 is formed from 4 data sets, each one with three instants of time: two instants before current one, previous and current.

- Option 3 is formed from 4 data sets, each one with four instants of time: three instants before current one, two instants before current one, previous and current.

Further details on the analyzed datasets can be found in Table 2 .

Table 2. Analyzed datasets.

\begin{tabular}{|c|c|c|c|c|c|}
\hline \multirow{2}{*}{$\frac{\text { Option }}{1}$} & \multirow{2}{*}{$\begin{array}{l}\mathrm{N}^{\mathrm{o}} \text { of columns } \\
2\end{array}$} & \multicolumn{4}{|c|}{ Columns (time instants) } \\
\hline & & & & $\mathrm{t}-1$ & $\mathrm{t}$ \\
\hline 2 & 3 & & $\mathrm{t}-2$ & $\mathrm{t}-1$ & $\mathrm{t}$ \\
\hline 3 & 4 & $t-3$ & $\mathrm{t}-2$ & $\mathrm{t}-1$ & $\mathrm{t}$ \\
\hline
\end{tabular}

So in this case, there is an array of 12 different combinations available to assemble the datasets for the tests: four different ways of summarizing the measures to construct each sample (summarizing 2, 4, 8 or 16) and three combinations for the number of dimensions considered in each sample ( $t-3$ to $t, t-2$ to $t$ and $t-1$ to $t$ ).

\subsection{Experimental Results}

Experiments have been performed with all datasets previously explained. All data were gathered when the volunteer performed the same three activities, but the data were summarized in different ways (See previous page). The purpose is therefore, to find which of those combinations for data gathering could be considered the best one to separate activities, in order to be used in further analyses. Experiments have been performed using the SOM as a classifier, but two other measures have been performed on the resulting map. A 10-fold cross-validation schema has been used in order to obtain the most significant results as possible. 


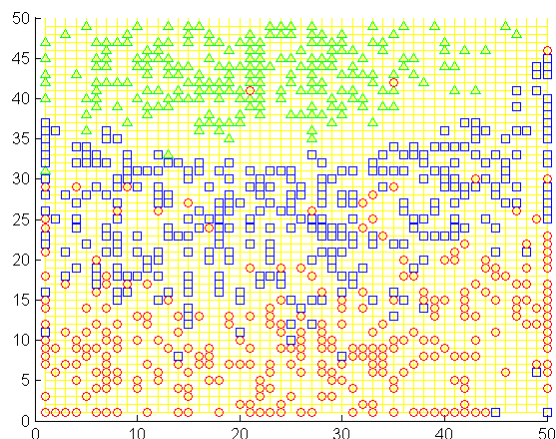

Figure 2a. 16 measurements and 4 dimensions in each sample ( $t-3$ to $t)$.

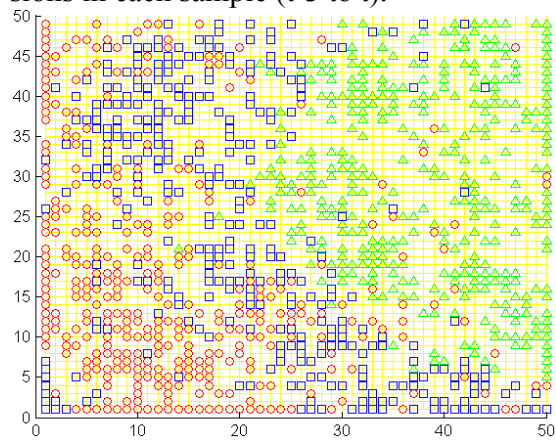

Figure 2c. 2 measurements and 4 dimensions in each sample ( $t-3$ to $t)$.

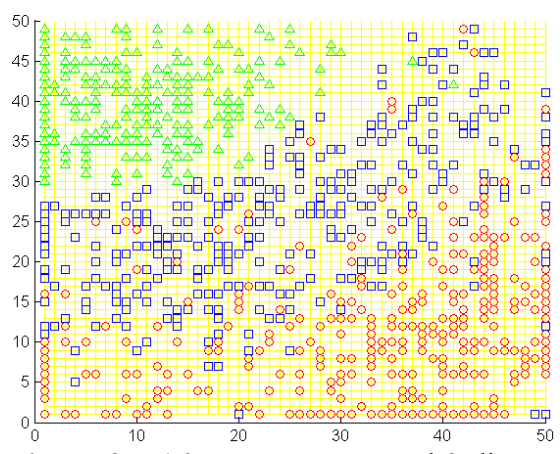

Figure 2b. 16 measurements and 2 dimensions in each sample ( $\mathrm{t}-1$ to $\mathrm{t})$.

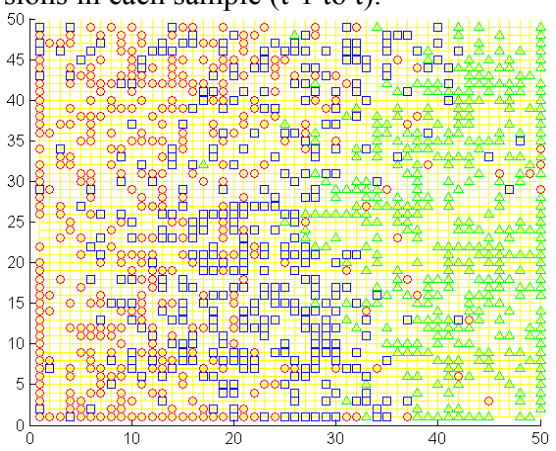

Figure 2d. 2 measurements and 2 dimensions in each sample ( $t-1$ to $t)$.

Figure 2. Different maps obtained for the most distinctive dataset combinations.

Figure 2 shows the maps obtained for each dataset. Each one of the three movements (classes) is depicted in a different way: green triangles, red circles and blue squares. As can be seen, maps obtained when summarizing samples in 16 measures a sample, yield maps separating in a clearer way the three different classes of the dataset. On the contrary, when using only 2 measurements to obtain a sample for the dataset, the classes in the maps appear more mixed. This is especially true for classes "walking" (red circle) and "walking holding a bag" (blue square). Also, the results can be considered better when 4 dimensions are used in each sample (Figure 2a) than when using only two (Figure 2b). In this case the third class ("walking with a hand in the pocket") can be easily separated from the other two, as there is a clear gap of blank space separating them. Also, although there is a certain overlapping between the other two classes in all cases, the organization seems to be more stratified when using 4 dimensions in each sample (Figure 2a). 


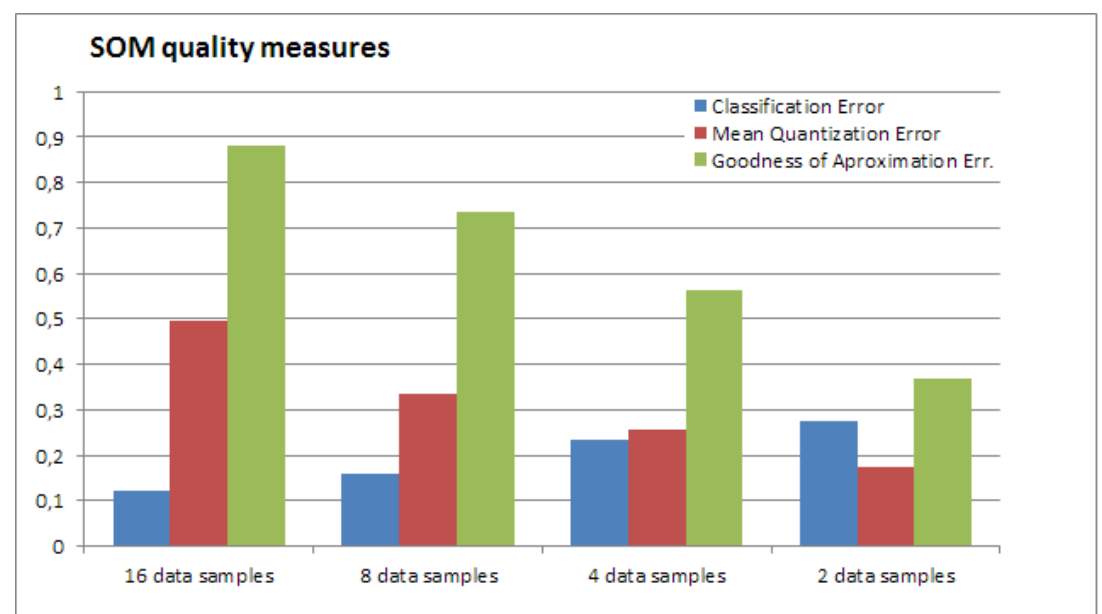

Figure 3. Comparison of the SOM quality obtained when varying the number of measures summarized in each sample.

Quantifiable results have been also obtained for each of the maps trained over the different datasets.

Firstly, a comparison of the three measures when modifying the frequency in which the data samples are considered is included in Figure 3 All measures represented are error measures; so the lower the value, the best the model is performing. The Classification error is a percentage measure, while the other two are dimensionless measures. As can be seen, the classification performance clearly degrades when reducing the number of measurements summarized in each data sample (from 16 to 2). The other two measures seems to improve with this reduction. This is due to the fact that the dataset becomes sparser when using more frequently acquired measurements, therefore, decreasing the quantization error of the maps. In the case of this study, this is not the desired effect: a situation where samples are clearly separated (i.e. distinguishable) is the preferred one. As Goodness of Approximation Error accounts also for data quantization error, it behaves in a very similar way to the MQE. 


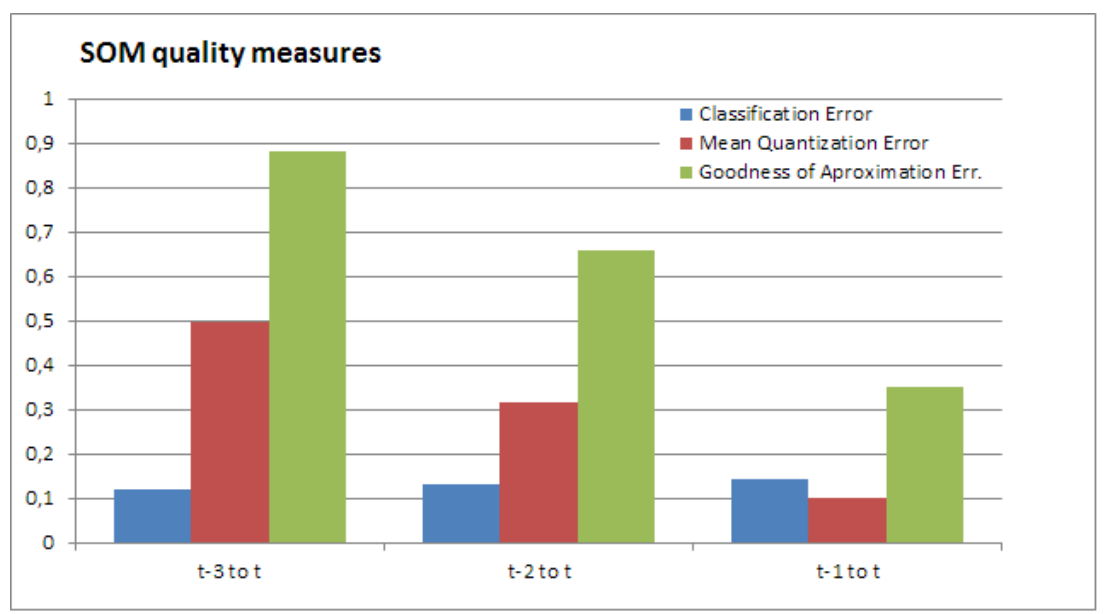

Figure 4. Comparison of the SOM quality obtained when varying the number of time instants considered for each sample.

Finally, the comparison of the results when considering the measures registered on 4,3 or 2 previous time instants is included in Figure 4. Although it is no as significantly as in the previous comparison, classification error increases when decreasing the number of dimensions used to characterize a sample (from 11\% with 4 dimensions to $14 \%$ with only 2 ). The other two measures decrease for similar reasons as explained before.

\section{Conclusions and Future Research Lines}

This work presents a first approximation to the problem of the automatic identification of the activities performed by a human user by means of a movement sensor. Several datasets have been registered in order to check which of the measuring conditions are the most appropriate to perform a simple identification of activities. Results point to the fact that the best settings are the ones that group more measures in each training sample (16 measures by sample in the experiments) as this tends to generate less sparse data in the data space, making classification easier. Also, including multiple dimensions to characterize each sample (4 dimensions in the experiments) in order to favour the correct classification; since the majority of automated learning algorithms rely on multi-dimensional analysis to perform their training.

Future work will be aimed at improving the classification results currently presented. Other automatic classification methods such as Artificial Neural Networks or Classification Trees could be used to further improve the results obtained by the SOM by constructing a hierarchical structure. 
Acknowledgments This research has been partially supported through the Spanish Ministry of Science and Innovation (MICINN) under project CIT-020000-2009-12 (funded by the European Regional Development Fund); project of the Spanish Ministry of Science and Innovation TIN2010-21272-C02-01 (funded by the European Regional Development Fund) and MICINN PID 560300-2009-11. The authors would also like to thank the vehicle interior manufacturer, Grupo Antolin Ingenieria S.A., within the framework of the MAGNO2008 - 1028.- CENIT Project also funded by the MICINN.

\section{References}

[1] Comparing Self-Organizing Maps. ICANN'96, volume 1112, 1996.

[2] Fernando Bação, Victor Lobo, and Marco Painho. Self-organizing maps as substitutes for kmeans clustering. In Proceedings of the 5th international conference on Computational Science - Volume Part III, ICCS'05, pages 476-483, Berlin, Heidelberg, 2005. Springer-Verlag.

[3] Oresti Banos, Miguel Damas, Hector Pomares, Alberto Prieto, and Ignacio Rojas. Daily living activity recognition based on statistical feature quality group selection. Expert Systems with Applications, 39(9):8013-8021, 72012.

[4] Nicola Bicocchi, Marco Mamei, and Franco Zambonelli. Detecting activities from body-worn accelerometers via instance-based algorithms. Pervasive and Mobile Computing, 6(4):482495, 82010.

[5] Yen-Ping Chen, Jhun-Ying Yang, Shun-Nan Liou, Gwo-Yun Lee, and Jeen-Shing Wang. Online classifier construction algorithm for human activity detection using a tri-axial accelerometer. Applied Mathematics and Computation, 205(2):849-860, 11/15 2008.

[6] Analog Devices. Adx1335, 2012. Accelerometer.

[7] D. Fuentes, L. Gonzalez-Abril, C. Angulo, and J. A. Ortega. Online motion recognition using an accelerometer in a mobile device. Expert Systems with Applications, 39(3):2461-2465, 2/15 2012.

[8] Yu-Jin Hong, Ig-Jae Kim, Sang Chul Ahn, and Hyoung-Gon Kim. Mobile health monitoring system based on activity recognition using accelerometer. Simulation Modelling Practice and Theory, 18(4):446-455, 42010.

[9] T. Kohonen. Self-Organizing Maps. Springer Series in Information Sciences. Springer, 2001.

[10] Paula Martiskainen, Mikko Järvinen, Jukka-Pekka Skön, Jarkko Tiirikainen, Mikko Kolehmainen, and Jaakko Mononen. Cow behaviour pattern recognition using a three-dimensional accelerometer and support vector machines. Applied Animal Behaviour Science, 119(1-2):3238, 62009.

[11] Rossana Muscillo, Maurizio Schmid, Silvia Conforto, and Tommaso D’Alessio. Early recognition of upper limb motor tasks through accelerometers: real-time implementation of a dtw-based algorithm. Computers in biology and medicine, 41(3):164-172, 32011.

[12] M Riedmiller and H Braun. A direct adaptive method for faster backpropagation learning: the rprop algorithm. IEEE International Conference on Neural Networks, 1(3):586-591, 1993.

[13] Javier Sedano, Camelia Chira, Jerónimo González, and José Ramón Villar. Intelligent system to measuring stress: Stresstic. DYNA, 87-3:336-344, 2012.

[14] Kristof Van Laerhoven and Ozan Cakmakci. What shall we teach our pants? 2000.

[15] Juha Vesanto. Data mining techniques based on the self-organizing map. Master's thesis, Helsinki University of Technology, May 1997.

[16] Jin Wang, Ronghua Chen, Xiangping Sun, Mary F. H. She, and Yuchuan Wu. Recognizing human daily activities from accelerometer signal. Procedia Engineering, 15(0):1780-1786, 2011. 
[17] Huikai Xie, Gary K. Fedder, and Robert E. Sulouff. 2.05 - accelerometers. In Editors in Chief: Yogesh Gianchandani, Osamu Tabata, and Hans Zappe, editors, Comprehensive Microsystems, pages 135 - 180. Elsevier, Oxford, 2008.

[18] Jhun-Ying Yang, Jeen-Shing Wang, and Yen-Ping Chen. Using acceleration measurements for activity recognition: An effective learning algorithm for constructing neural classifiers. Pattern Recognition Letters, 29(16):2213-2220, 12/1 2008. 